\title{
A cross-sectional analysis of registered clinical trials on the use of particle beam radiation therapy in head and neck cancers
}

\author{
Wenna Zhang ${ }^{1,2}$, Lin Kong ${ }^{2,3}$, Jiade J. Lu ${ }^{1,2}$ \\ ${ }^{1}$ Department of Radiation Oncology, Shanghai Proton and Heavy Ion Center, Shanghai, China; ${ }^{2}$ Shanghai Engineering Research Center of Proton \\ and Heavy Ion Radiation Therapy, Shanghai, China; ${ }^{3}$ Department of Radiation Oncology, Shanghai Proton and Heavy Ion Center, Fudan University \\ Cancer Hospital, Shanghai, China \\ Contributions: (I) Conception and design: JJ Lu, W Zhang; (II) Administrative support: L Kong; (III) Provision of study materials or patients: W \\ Zhang; (IV) Collection and assembly of data: W Zhang; (V) Data analysis and interpretation: W Zhang, JJ Lu; (VI) Manuscript writing: All authors; \\ (VII) Final approval of manuscript: All authors. \\ Correspondence to: Lin Kong; Jiade J. Lu. Shanghai Proton and Heavy Ion Center, 4365 Kangxin Road, Shanghai, China. \\ Email: lin.kong@sphic.org.cn; jiade.lu@sphic.org.cn.
}

\begin{abstract}
Background: To present and analyze the current status of registered clinical trials on particle beam (including proton and carbon ion beam) radiation therapy (PBRT) for head and neck (H\&N) malignancies, and to provide insights for future clinical research, we designed the cross-sectional analysis.

Methods: We identified and analyzed all clinical trials of interest registered on ClinicalTrials.gov and PTCOG.ch until March 22, 2020.

Results: We identified 57 registered clinical trials related to the use of proton therapy or carbon ion radiation therapy (CIRT) in H\&N malignancies. There were 20, 27, and 5 trials focused on CIRT, proton therapy, and both ions, respectively. The eligible trials were registered between 2007 and 2020, mainly focused on adenoid cystic carcinoma (ACC), squamous cell carcinoma (SCC), sinonasal malignancies (SNM), skull base tumors, locally advanced, and recurrent tumors. The nature of 23 (40\%) trials were not stated and could not be identified. A total of 25 (44\%) registered trials were phase II, including randomized controlled trials (RCTs). There were 14 RCTs (7 phase II, 2 phase II/III, 2 phase III, 1 phase I/II, and 2 phase not applicable), and 25 studies including RCTs were registered before the first enrolment. There were 11 completed clinical trials among the eligible trials, including 7 with published trial-related results.

Conclusions: Less than $10 \%$ of the countries with PBRT treatment facilities in operation have initiated clinical trials on $\mathrm{H} \& \mathrm{~N}$ cancer. Furthermore, among all registered trials, less than $10 \%$ have been completed with results published. More clinical trials, especially high quality trials, are needed for optimizing and standardizing treatment techniques of PBRT for H\&N malignancies.
\end{abstract}

Keywords: Registered clinical trial; particle beam radiation therapy (PBRT); proton therapy; carbon ion radiation therapy (CIRT); head and neck cancer

Submitted Aug 17, 2020. Accepted for publication Mar 12, 2021.

doi: $10.21037 /$ atm-20-7760

View this article at: https://dx.doi.org/10.21037/atm-20-7760

\section{Introduction}

According to the 2018 global cancer statistics, head and neck $(\mathrm{H} \& \mathrm{~N})$ cancer was ranked the seventh most common type of cancer, with 890,000 new cases and 450,000 deaths worldwide $(1,2)$. Long-term survival can be achieved in $70-90 \%$ of patients in the early stage after local therapy, including surgery or radiation therapy alone $(2,3)$. However, $15-40 \%$ of patients with locally advanced diseases develop local recurrence. With a high rate of distant failure, the 5 -year overall survival (OS) rates remain suboptimal for these patients, at approximately $50 \%$ (4). Furthermore, $56 \%$ of patients who survive beyond 3 years eventually die 
of recurrence, second primary cancers, or other cancerrelated causes within 10 years $(5,6)$. For patients who fail locally after definitive radiotherapy, re-irradiation is one of the most important salvage treatment options despite being limited by treatment-associated toxicities. In addition, improved quality of life after high dose radiotherapy is of significant concern for long-term survivors. Clearly, more effective and safer radiation technologies are needed for $\mathrm{H} \& \mathrm{~N}$ cancer patients, especially those presenting with locally advanced disease.

Harnessing the unique physical characteristics of the Bragg peak, particle beam radiotherapy results in minimal radiation doses to organs at risk (OARs). Ultimately, this minimizes radiation-related adverse reactions while maintaining therapeutic doses to tumor targets, thereby improving the therapeutic ratio (7). Furthermore, the higher linear energy transfer (LET) and relative biological effectiveness (RBE) of heavy-ions (i.e., carbon ions) are more effective, at least in theory, for radioresistant malignancies such as soft-tissue sarcoma or tumors in a hypoxic environment (8). Most of the particle beam radiation therapy (PBRT) facilities are located in the United States and Japan, and are equipped with proton treatment systems. Globally, 12 heavy ion therapy centers for clinical application are equipped with carbon ion treatment systems. As a novel technology in cancer radiation therapy, a clinical trial is the most appropriate method to optimize treatment strategies and standards. High quality data from prospective clinical trials, particularly randomized controlled trials (RCTs), are needed to change the current treatment standards in PBRT. Nevertheless, there are very limited prospective trials and no current results from RCTs available on PBRT for $\mathrm{H} \& \mathrm{~N}$ cancers. Thus, the aim of this analysis is to determine the current status of registered clinical trials on the use of proton therapy and carbon ion radiation therapy (CIRT) in H\&N cancers, providing insight for designing future clinical research.

\section{Methods}

We searched for clinical trials of interest registered on the ClinicalTrials.gov database and the Particle Therapy CoOperative Group (PTCOG) database (last search updated March 22, 2020).

\section{Selection of clinical trials and literature search}

We identified the clinical trials listed in the $\mathrm{H} \& \mathrm{~N}$ cancer part of the PTCOG website (https://www.ptcog.ch/index. $\mathrm{php} /$ clinical-protocols) (last updated on January 3, 2019). Four retrospective studies from Japan were registered in the University Hospital Medical Information Network Clinical Trials Registry (UMIN-CTR) (https://www.umin.ac.jp/ ctr/), and were collected by the PTCOG, thus meeting our inclusion criteria. We then did a systematic search of clinical trials on ClinicalTrials.gov (https://clinicaltrials.gov) (last search updated in March 22, 2020 by the authors) using the following procedure: For trials on CIRT, we searched with the phrase "carbon ion" (48 items retrieved) directly; for trials on proton therapy, we searched with the phrases "head and neck" and "proton" (78 items retrieved); for trials on skull base tumors, we searched with the phrases "skull base" and "proton" (11 items retrieved). We identified publications by searching the registration number and official titles in PubMed. All trials were examined to avoid duplication prior to further analysis.

\section{Inclusion and exclusion criteria}

We included clinical trials that met all of the following criteria: (I) patients had a primary $\mathrm{H} \& \mathrm{~N}$ malignancy (i.e., nasal and paranasal sinuses, oral cavity, pharynx, larynx, tongue, lip, salivary glands, eyelid, palate, tonsil, ear, nose, neck, and skull base); (II) patients were treated by proton therapy and/or CIRT.

We excluded clinical trials if: (I) the condition/diseases of the clinical trials were tumors beyond $\mathrm{H} \& \mathrm{~N}$; (II) the condition/diseases focused on tumors from the whole body; (III) the indexed condition/disease was thyroid cancer, esophageal cancer (including cervical esophagus), or malignant lymphoma because of their distinct treatment planning from typical $\mathrm{H} \& \mathrm{~N}$ cancers; (IV) the technology of radiotherapy was not extractable.

\section{Data collection}

Data from eligible clinical trials were extracted and checked by two independent reviewers, with a third reviewer who helped reach consensuses and examined discrepancies. General characteristics including the registration number, country, sponsor, condition/diseases, phase, intervention, radiotherapy type, sample size, randomization status, masking, study design, primary outcome measures, secondary outcome measures, study start date, age, sex, registration date, last update posted date, recruitment status, publications, and titles were recorded. 


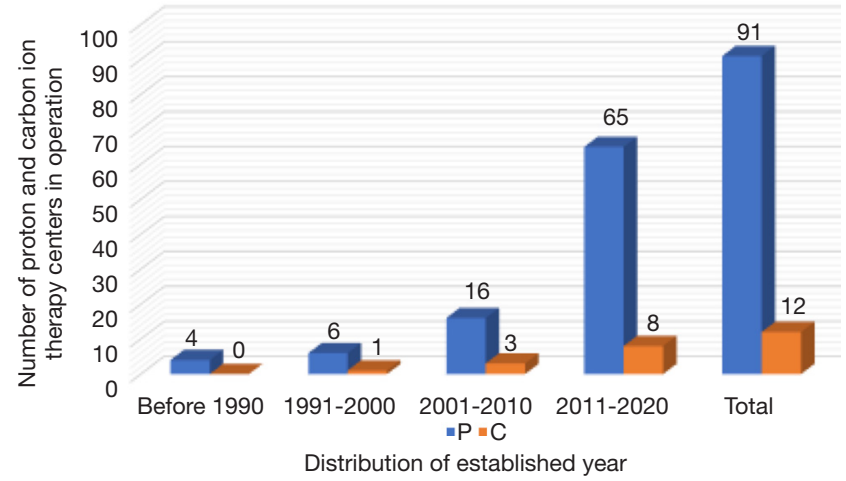

Figure 1 Distribution of proton and carbon ion therapy centers in operation by established year of commencement. $\mathrm{P}$, proton therapy center; C, carbon ion therapy center.

\section{Data analysis}

We recorded and classified the collected data and displayed the analysis in three parts: (I) geographic distribution of the eligible clinical trials; (II) characteristics of the eligible clinical trials; (III) failure-cause analysis of the non-active clinical trials.

\section{Results}

We identified 57 registered clinical trials (27 on proton therapy, 25 on CIRT, and 5 on both) on H\&N cancers from 9 countries and 20 sponsors.

\section{Geographic distribution of the eligible clinical trials}

The types of particles that have been used in clinical practice include proton and carbon ions. According to the PTCOG (updated in February 2020), 101 proton or carbon ion facilities are currently in operation globally, with 65 proton and 8 carbon therapy centers commencing operation in the past decade (Figure 1). However, only 20 institutions (of 9 countries) have registered clinical trials on $\mathrm{H} \& \mathrm{~N}$ cancers. The distribution of eligible clinical trials by sponsors and particle types is detailed in Table 1. Among the eligible clinical trials, there were 30 CIRT trials (30/57, $52.6 \%$ ) from Germany [10], Japan [10], China [6], Italy [3], and the Netherlands [1]. The CIRT section of the clinical trials registered by the Netherlands were conducted in collaboration with Germany. Out of the 27 registered trials on proton therapy, 21 were from the USA. Heidelberg Ion Therapy Center (HIT) of the University of Heidelberg $(\mathrm{n}=9)$, Shanghai Proton and Heavy Ion Center (SPHIC) $(\mathrm{n}=6)$, and M.D. Anderson Cancer Center (MDACC) $(\mathrm{n}=6)$ were the top 3 centers that had registered clinical trials related to the application of PBRT in H\&N malignancies. Denmark, Sweden and Korea each had 1 registered proton therapy clinical trial on $\mathrm{H} \& \mathrm{~N}$ cancer.

\section{Characteristics of the eligible clinical trials}

The nature of the trial, indexed disease/condition, recruitment/performance status, and status of publications are detailed in Table 2. There were 57 clinical trials on $\mathrm{H} \& \mathrm{~N}$ cancers, including 8 trials for skull base tumors. The first two posted clinical trials focused on skull base chondrosarcoma and chordoma, and were both registered on July 4, 2007 by researchers from MDACC. A total of 41 trials were registered in the past decade, including 29 trials (29/57, 50.8\%) registered between 2016 and 2020, and 12 (12/57, 21.0\%) registered between 2011 and 2015. These eligible trials mainly focused on patients diagnosed with adenoid cystic carcinoma (ACC), squamous cell carcinoma (SCC), sinonasal malignancies (SNM), skull base tumors of various types, and locally advanced or recurrent tumors in adult patients ( $\geq 18$ years old, regardless of gender). Most of the trials were designed with small sample sizes, and only 16 trials had over 100 estimated or actual enrolled participants.

There were 25 (43.86\%) phase II trials [including phase $\mathrm{I} / \mathrm{II}(\mathrm{n}=5)$ and phase II/III $(\mathrm{n}=2)$ trials]. There were 2 phase III RCTs. The phases of 23 studies $(40.35 \%)$ could not be identified/determined. There were 19 observational cohorts (5 from Japan and 9 from the USA) and 38 interventional clinical trials. In terms of intervention study designs, 21 trials had parallel assignment and 30 trials had single group assignment. Furthermore, there were 14 RCTs ( 7 phase II; 2 phase II/III; 2 phase III; 1 phase I/II; 2 phase not applicable).

A total of 25 studies including RCTs were registered before their first enrollment, however, all the trials were open label. There were 11 completed clinical trials and 17 recruiting trials. However, only 7 trials had published the trial-related interim data or final results, and 28 trials updated the status within 1 year.

\section{Analysis of the non-active clinical trials}

There were 46 unfinished and 11 completed clinical trials. Trials were defined as "active" if they had been updated or 
Table 1 Geographic distribution of the eligible clinical trials

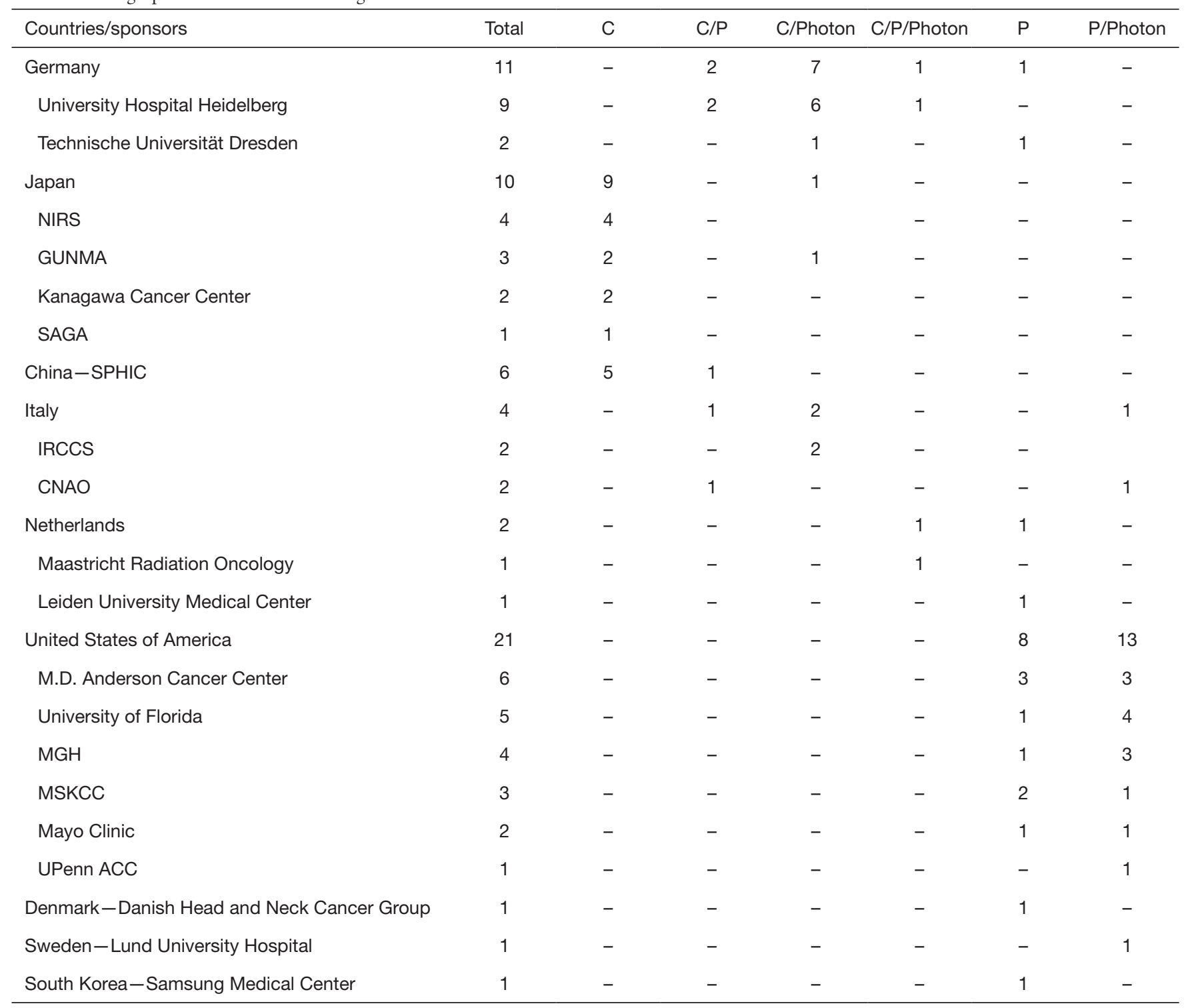

C, carbon ion radiation therapy; P, proton therapy; Photon, photon-based radiation therapy; NIRS, National Institute of Radiological Science; GUNMA, Gunma University Heavy Ion Medical Center; SAGA, SAGA HIMAT Foundation; SPHIC, Shanghai Proton and Heavy lon Center; CNAO, National Center of Oncological Hadrontherapy; MGH, Massachusetts General Hospital; MSKCC, Memorial Sloan-Kettering Cancer Center; UPenn ACC, Abramson Cancer Center of the University of Pennsylvania.

had interim results published within 3 years, and the rest were classified as "non-active". According to these criteria, there were 31 active trials and 15 non-active trials. Through further analysis of the 15 non-active trials, we found that the 2 phase III RCTs with an estimated sample size over 100 were both included (phases of other trials: 6 phase II; 2 phase I/II; 5 phase not stated), and 6 trials were terminated because of slow enrollment. There were 12 carbon ion and
3 proton therapy non-active trials from HIT [5], University of Florida [3], SPHIC [2], Gunma University Heavy Ion Medical Center [2], Kanagawa Cancer Center [2], and the SAGA HIMAT Foundation [1]. Moreover, 11 trials were interventional and 4 trials were observational, focusing on unspecified $\mathrm{H} \& \mathrm{~N}$ cancers [3], nasopharyngeal carcinoma [3], chondrosarcoma of the skull base [2], malignant melanoma [2], sarcoma [1], skin cancer [1], oropharyngeal cancer [1], 
Table 2 Characteristics of the eligible clinical trials

\begin{tabular}{|c|c|c|c|c|c|c|c|c|c|c|}
\hline Characteristics & Total & Germany & Japan & China & Italy & $\begin{array}{l}\text { The } \\
\text { Netherlands }\end{array}$ & USA & Denmark & Sweden & $\begin{array}{l}\text { South } \\
\text { Korea }\end{array}$ \\
\hline No. of trials in total & 57 & 11 & 10 & 6 & 4 & 2 & 21 & 1 & 1 & 1 \\
\hline \multicolumn{11}{|l|}{ Conditions/diseases } \\
\hline Adenoid cystic carcinoma & 4 & 2 & 1 & 1 & - & - & - & - & - & - \\
\hline Sarcoma & 1 & - & 1 & - & - & - & - & - & - & - \\
\hline Nasopharyngeal carcinoma & 2 & - & - & - & - & - & 2 & - & - & - \\
\hline Salivary gland tumor & 1 & 1 & - & - & - & - & - & - & - & - \\
\hline Recurrent tumor & 9 & 1 & - & 5 & - & 1 & 2 & - & - & - \\
\hline Skull base tumor & 8 & 3 & - & - & - & - & 5 & - & - & - \\
\hline Mixed/other & 9 & - & 1 & - & 2 & - & 3 & 1 & 1 & 1 \\
\hline \multicolumn{11}{|l|}{ Phase } \\
\hline $\mathrm{I} / \mathrm{II}$ & 5 & 1 & - & 3 & - & - & 1 & - & - & - \\
\hline$\|$ & 25 & 6 & 3 & 1 & 4 & - & 9 & 1 & - & 1 \\
\hline II/III & 2 & - & - & 1 & - & - & 1 & - & - & - \\
\hline $\mathrm{N} / \mathrm{A}$ & 6 & - & 5 & - & - & - & 1 & - & - & - \\
\hline \multicolumn{11}{|l|}{ Randomization status } \\
\hline Randomized & 14 & 6 & - & 2 & - & - & 5 & - & 1 & - \\
\hline Non-randomized & 34 & 4 & 5 & 4 & 2 & 2 & 15 & 1 & - & 1 \\
\hline $\mathrm{N} / \mathrm{A}$ & 9 & 1 & 5 & - & 2 & - & 1 & - & - & - \\
\hline \multicolumn{11}{|l|}{ No. of enrolled participants } \\
\hline Less than 100 & 41 & 7 & 8 & 5 & 4 & 2 & 13 & 1 & - & 1 \\
\hline More than 100 & 16 & 4 & 2 & 1 & - & - & 8 & - & 1 & - \\
\hline \multicolumn{11}{|l|}{ Study design } \\
\hline Interventional clinical trial & 38 & 10 & 5 & 5 & 3 & - & 12 & 1 & 1 & 1 \\
\hline Observational cohort & 19 & 1 & 5 & 1 & 1 & 2 & 9 & - & - & - \\
\hline
\end{tabular}

Table 2 (continued) 
Table 2 (continued)

\begin{tabular}{|c|c|c|c|c|c|c|c|c|c|c|}
\hline Characteristics & Total & Germany & Japan & China & Italy & $\begin{array}{c}\text { The } \\
\text { Netherlands }\end{array}$ & USA & Denmark & Sweden & $\begin{array}{l}\text { South } \\
\text { Korea }\end{array}$ \\
\hline \multicolumn{11}{|l|}{ Registration time } \\
\hline After enrollment & 32 & 3 & 4 & 3 & 3 & 1 & 17 & - & 1 & - \\
\hline 2011-2015 & 12 & 2 & 3 & 1 & 2 & 1 & 3 & - & - & - \\
\hline $2016-2020$ & 29 & 3 & 7 & 5 & 2 & 1 & 8 & 1 & 1 & 1 \\
\hline \multicolumn{11}{|l|}{ Last update posted } \\
\hline Completed & 11 & - & 5 & - & 1 & 1 & 4 & - & - & - \\
\hline Recruiting & 17 & 4 & 1 & 1 & - & - & 10 & - & 1 & - \\
\hline Not yet recruiting & 11 & 3 & 2 & 2 & 1 & 1 & - & 1 & - & 1 \\
\hline Active, not recruiting & 7 & - & - & 1 & 2 & - & 4 & - & - & - \\
\hline Terminated & 8 & 1 & 2 & 2 & - & - & 3 & - & - & - \\
\hline Unknown & 3 & 3 & - & - & - & - & - & - & - & - \\
\hline \multicolumn{11}{|l|}{ Status of trials } \\
\hline
\end{tabular}

USA, United States of America; N/A, not applicable.

SNM [1], and salivary gland tumors [1].

\section{Discussion}

The use of proton therapy and CIRT has prevailed in the management of $H \& N$ malignancies, including those originating from the skull base, for the past several decades. However, there appeared to be insufficient clinical studies, especially prospective clinical trials initiated in countries with currently operating PBRT facilities. Despite the doubling of the number of registered studies during the past decade, only half of the countries (9/18) with PBRT facilities have registered clinical trials for $\mathrm{H} \& \mathrm{~N}$ malignancies. In addition, the registration of trials appeared to be sluggish, as more than $50 \%$ (32 of 57 trials, $56.1 \%$ ) were not registered before the first enrollment. This phenomenon is consistent with the results of a previous study for prospective clinical trials of the other specialties (9). Furthermore, follow-through of clinical trial registration seems to be a concern, as less than half of the registered trials $(28 / 57,49.1 \%)$ were posted or have been updated within the past year. In addition, only 7 of the 57 registered studies were published (8.8\%) since 2007, when the first trials on PBRT for H\&N malignancies were registered. Most of the published studies were retrospective or not directly from the results of a prospective trial. Clearly, for 
a new technology such as PBRT that has not accumulated much clinical evidence and experience, it is important to strengthen the registration and timely update of trial statuses so that researchers and clinicians can reference the knowledge and the protocols of the ongoing trials.

The biological differences between various types of ionizing radiation beams, namely photon, proton, and carbon ion beams, are of great interest. Basically, proton and carbon ion radiotherapy can fit all kinds of tumors, but it depends on different primary sites, pathological types, radiation course and so on to make the best treatment regimen. There were 15 trials designed to compare the adverse effects or treatment outcomes between proton versus photon therapies, and 4 trials to compare proton versus carbon ion therapies. Centers in the United States have registered 13 clinical trials to compare proton with photon therapies for treating H\&N cancers. However, so far there is only 1 article studying oropharyngeal squamous carcinoma (OPC) published in 2016 (10), which is a part of the phase II/III RCT (NCT01893307) initiated in 2013 comparing intensive modulated proton therapy (IMPT) versus intensive modulated radiotherapy (IMRT). Only the results of 50 patients treated with IMPT were reported. There were no severe toxicities observed, with acute mucositis (58\%) and late dysphagia (12\%) the most common grade 3 acute toxicity and late toxicity, respectively. The 2-year OS and progression free survival (PFS) rates were $94.5 \%$ and $88.6 \%$ respectively, with a median follow-up time of 29 months. The 4 registered trials comparing proton therapy with carbon ion therapy were from Germany [2], China [1], and Italy [1], but all without published results.

Among the 30 eligible CIRT clinical trials, 6 studied recurrent tumors, 4 studied ACC, 4 studied skull base tumors, 3 studied SNM, and 3 studied mucosal melanoma. Trials on CIRT for recurrent tumors originated mainly from China (SPHIC), which is equipped with both proton therapy and CIRT facilities. However, 8 trials among the 30 clinical trials were terminated for slow enrollment or other reasons, resulting in 15 non-active trials. There were 7 published trial protocols out of 11 registered clinical trials from HIT in Germany. However, only 2 articles were published based on 2 clinical trials titled COSMIC and ACCEPT (11). The published COSMIC (12) trial presented the interim results of a prospective phase II study on 29 patients with salivary gland tumors. After the completion of IMRT (50 Gy in 25 fractions) and carbon ion boost (24 Gy RBE in 8 fractions), the occurrence of grade 3 mucositis (National
Cancer Institute Common Toxicity Criteria v3.0) was $25 \%$ in the $\mathrm{R} 2$ resection group and $15.4 \%$ in the $\mathrm{R} 1$ resection group $(\mathrm{P}>0.05)$. The results indicated the safety of the combined treatment, and patients with macroscopically complete resection and gross residual tumors had similar toxicities. Ten articles (13-22) were published from PBRT centers in Japan based on their registered studies, and most were retrospective. The studies were registered on UMINCTR shortly before the publication of the papers (date of registration in relation to publication not shown in our analysis). The Japan Carbon-Ion Radiation Oncology Study Group (J-CROS) Study registered in 2016 was a database of 1,000 H\&N cancer patients treated with CIRT. Results of 7 retrospective analyses were published from this single database with different subgroup populations focused on ACC (22), SNM (16), mucosal melanoma (17), olfactory neuroblastoma (21), non-SCC of the nasopharynx (13), external auditory canal and middle ear carcinoma (14), and mucoepidermoid carcinoma (19).

It is difficult to initiate and carry out RCTs to directly compare the efficacy or adverse effects between regimens using different radiation beams (e.g., photon versus carbon ion beams). A number of reasons contribute to this difficulty. Firstly, few centers are equipped with photon, proton, as well as carbon ion radiotherapy equipment to facilitate RCTs to compare the clinical outcomes from various radiation technologies. Although international collaboration could support trials for investigators who have no direct access to PBRT equipment, the financial resources required for supporting such endeavors are usually vast and not practical, especially for RCTs with large sample sizes. In addition, patients with financial resources usually present with a direct request to be treated with more advanced radiation technologies, namely PBRT, and are not willing to be randomized to a conventional technology. Additionally, bias of patients towards clinical trials and wanting to avoid becoming a "guinea pig" is another reason for difficulty in the initiation and carrying out of PBRT clinical trials.

Our study is the first investigation to analyze the current status of clinical studies that focus on the use of PBRT for $\mathrm{H} \& \mathrm{~N}$ malignancies, as well as the current barriers in promoting the effective development of clinical standards for the novel technology. However, our analysis has several limitations. Firstly, although we have performed a thorough search using the ClinicalTrials.gov and the PTCOG databases, it is highly probable that missed clinical trials approved by the institutional review boards but not posted online affected the accuracy of our search results. Secondly, 
the interpretation of the results was based on posted and published data only. Communication with individual centers to verify the completeness, accuracy, and the timeliness of the studies was not conducted. We hope that more close collaboration between PBRT centers in the future could facilitate updating trial information more effectively.

\section{Conclusions}

The major concerns of clinical investigations on PBRT for $\mathrm{H} \& \mathrm{~N}$ malignancies include low registration rates, low publication rates, few RCTs, and high termination rates. Less than $10 \%$ of the countries with proton or carbon ion facilities in operation have initiated clinical trials on $\mathrm{H} \& \mathrm{~N}$ malignancies, and only 1 in 8 registered studies, which were mostly retrospective, have published results in peer-reviewed journals. Publications from the results of prospective clinical trials on the treatment of $H \& N$ malignancies using PBRT are limited. Clearly, more prospective clinical trials and their completed and published results are needed to optimize the treatment protocols and practice of PBRT for H\&N malignancies.

\section{Acknowledgments}

Funding: This study was supported by grants from Science and Technology Commission of Shanghai Municipality (Project No. 19411951000).

\section{Footnote}

Conflicts of Interest: All authors have completed the ICMJE uniform disclosure form (available at https://atm. amegroups.com/article/view/10.21037/atm-20-7760/coif). The authors have no conflicts of interest to declare.

Ethical Statement: The authors are accountable for all aspects of the work in ensuring that questions related to the accuracy or integrity of any part of the work are appropriately investigated and resolved.

Open Access Statement: This is an Open Access article distributed in accordance with the Creative Commons Attribution-NonCommercial-NoDerivs 4.0 International License (CC BY-NC-ND 4.0), which permits the noncommercial replication and distribution of the article with the strict proviso that no changes or edits are made and the original work is properly cited (including links to both the formal publication through the relevant DOI and the license). See: https://creativecommons.org/licenses/by-nc-nd/4.0/.

\section{References}

1. Bray F, Ferlay J, Soerjomataram I, et al. Global cancer statistics 2018: GLOBOCAN estimates of incidence and mortality worldwide for 36 cancers in 185 countries. CA Cancer J Clin 2018;68:394-424.

2. Chow LQM. Head and Neck Cancer. N Engl J Med 2020;382:60-72.

3. Pfister DG, Spencer S, Brizel DM, et al. Head and neck cancers, Version 2.2014. Clinical practice guidelines in oncology. J Natl Compr Canc Netw 2014;12:1454-87.

4. Braakhuis BJ, Brakenhoff RH, Leemans CR. Treatment choice for locally advanced head and neck cancers on the basis of risk factors: biological risk factors. Ann Oncol 2012;23 Suppl 10:x173-177.

5. Baxi SS, Pinheiro LC, Patil SM, et al. Causes of death in long-term survivors of head and neck cancer. Cancer 2014;120:1507-13.

6. Cohen EE, LaMonte SJ, Erb NL, et al. American Cancer Society Head and Neck Cancer Survivorship Care Guideline. CA Cancer J Clin 2016;66:203-39.

7. Wang W, Huang Z, Sheng Y, et al. RBE-weighted dose conversions for carbon ionradiotherapy between microdosimetric kinetic model and local effect model for the targets and organs at risk in prostate carcinoma. Radiother Oncol 2020;144:30-6.

8. Fossati P, Molinelli S, Matsufuji N, et al. Dose prescription in carbon ion radiotherapy: a planning study to compare NIRS and LEM approaches with a clinically-oriented strategy. Phys Med Biol 2012;57:7543-54.

9. Harriman SL, Patel J. When are clinical trials registered? An analysis of prospective versus retrospective registration. Trials 2016;17: 187.

10. Gunn GB, Blanchard P, Garden AS, et al. Clinical Outcomes and Patterns of Disease Recurrence After Intensity Modulated Proton Therapy for Oropharyngeal Squamous Carcinoma. Int J Radiat Oncol Biol Phys 2016;95:360-7.

11. Adeberg S, Akbaba S, Lang K, et al. The Phase 1/2 ACCEPT Trial: Concurrent Cetuximab and Intensity Modulated Radiation Therapy with Carbon Ion Boost for Adenoid Cystic Carcinoma of the Head and Neck. Int J Radiat Oncol Biol Phys 2020;106:167-73.

12. Jensen AD, Nikoghosyan AV, Lossner K, et al. IMRT and carbon ion boost for malignant salivary gland tumors: 
interim analysis of the COSMIC trial. BMC Cancer 2012;12:163.

13. Abe T, Ohno T, Koto M, et al. A multi-institutional retrospective study of carbon-ion radiotherapy for nonsquamous cell malignant tumors of the nasopharynx: Subanalysis of Japan Carbon-Ion Radiation Oncology Study Group study 1402 HN. Cancer Med 2018;7:6077-83.

14. Hayashi K, Koto M, Demizu Y, et al. A retrospective multicenter study of carbon-ion radiotherapy for external auditory canal and middle ear carcinomas. Cancer Med 2019;8:51-7.

15. Ikawa H, Koto M, Hayashi K, et al. Feasibility of carbonion radiotherapy for oral non-squamous cell carcinomas. Head Neck 2019;41:1795-803.

16. Koto M, Demizu Y, Saitoh JI, et al. Definitive CarbonIon Radiation Therapy for Locally Advanced Sinonasal Malignant Tumors: Subgroup Analysis of a Multicenter Study by the Japan Carbon-Ion Radiation Oncology Study Group (J-CROS). Int J Radiat Oncol Biol Phys 2018;102:353-61.

17. Koto M, Demizu Y, Saitoh JI, et al. Multicenter Study of Carbon-Ion Radiation Therapy for Mucosal Melanoma of the Head and Neck: Subanalysis of the Japan Carbon-Ion Radiation Oncology Study Group (J-CROS) Study (1402
HN). Int J Radiat Oncol Biol Phys 2017;97:1054-60.

18. Naganawa K, Koto M, Takagi R, et al. Long-term outcomes after carbon-ion radiotherapy for oral mucosal malignant melanoma. J Radiat Res 2017;58:517-22.

19. Shirai K, Koto M, Demizu Y, et al. Multi-institutional retrospective study of mucoepidermoid carcinoma treated with carbon-ion radiotherapy. Cancer Sci 2017;108:1447-51.

20. Shirai K, Saitoh JI, Musha A, et al. Prospective observational study of carbon-ion radiotherapy for nonsquamous cell carcinoma of the head and neck. Cancer Sci 2017;108:2039-44.

21. Suefuji H, Koto M, Demizu Y, et al. A Retrospective Multicenter Study of Carbon Ion Radiotherapy for Locally Advanced Olfactory Neuroblastomas. Anticancer Res 2018;38:1665-70.

22. Sulaiman NS, Demizu Y, Koto M, et al. Multicenter Study of Carbon-Ion Radiation Therapy for Adenoid Cystic Carcinoma of the Head and Neck: Subanalysis of the Japan Carbon-Ion Radiation Oncology Study Group (J-CROS) Study (1402 HN). Int J Radiat Oncol Biol Phys 2018;100:639-46.

(English Language Editor: C. Betlazar-Maseh)
Cite this article as: Zhang W, Kong L, Lu JJ. A cross-sectional analysis of registered clinical trials on the use of particle beam radiation therapy in head and neck cancers. Ann Transl Med 2022;10(22):1192. doi: 10.21037/atm-20-7760 